EPJ Web of Conferences 18, 04001 (2011)

DOI: $10.1051 /$ epjconf/20111804001

(C) Owned by the authors, published by EDP Sciences, 2011

\title{
Croissance des HAP et des suies et transition phase gaz-phase solide dans les processus de combustion
}

\author{
P. Desgroux, A. El Bakali et X. Mercier \\ Physico-Chimie des Processus de Combustion et de l'Atmosphère (PC2A, UMR CNRS \\ 8522) Bâtiment C11, Université Lille 1, 59655 Villeneuve d'Ascq Cedex, France
}

\begin{abstract}
This article is addressed to a scientific community familiar to astrophysics, and most researchers are not familiar with combustion science. Thus the goal is to provide a basic understanding of "the flame" and the different steps that lead to soot particle formation in conditions that are generally close to atmospheric pressure and high temperature, i.e. in conditions far from those encountered in most astrophysical media. Some experimental techniques of sooty flame investigations will also be introduced. The main aim is to outline the concepts and the tools of combustion science to facilitate fruitful scientific exchanges between two communities that are motivated by the understanding of the formation and evolution of PAHs and soot-like particles, in completely different environments. It should be noted that the description does not pretend to be exhaustive at all and certain theoretical and experimental approaches are not described nor even mentioned.
\end{abstract}

Résumé. Cet article s'adresse à une communauté «astrophysique» non familière avec la combustion. Il a pour but de donner quelques bases permettant de comprendre «la flamme» et les étapes chimiques principales conduisant à la formation de particules de suie dans un milieu généralement à pression atmosphérique et haute température, c'est-à-dire dans des conditions de pression et température très éloignées de celles rencontrées dans le milieu interstellaire. Certaines méthodes expérimentales d'investigation de flammes suitées sont également présentées. L'objectif étant de pouvoir jeter des ponts entre deux communautés éloignées mais rassemblées par un même intérêt pour les hydrocarbures aromatiques polycycliques et les particules de suie. Il est clair que la présentation qui est faite ici est très succincte et qu'elle fait l'impasse sur certaines théories, approches, méthodes expérimentales...

\section{QUELQUES NOTIONS SUR LA COMBUSTION ET LES FLAMMES}

La combustion est un des principaux moyens de conversion d'énergie. Elle est utilisée dans un grand nombre de systèmes pour produire de la chaleur (chaudières, fours), de l'électricité (centrales thermiques) et pour le transport (moteurs automobiles, aéronautiques, fusée).

En première approche, la combustion peut être caractérisée comme une réaction irréversible fortement exothermique entre un combustible et un comburant selon le schéma global :

$$
\text { combustible + comburant } \quad \Longrightarrow \text { produits de combustion + chaleur }
$$

Cette réaction induit un fort dégagement de chaleur qui se produit dans une zone très mince de la flamme (appelée zone réactionnelle) où le gradient thermique est très élevé. Le taux de réaction suit une loi d'Arrhenius.

Lorsque la combustion du mélange conduit à la consommation totale du combustible et du comburant et à la formation des produits dans leur état d'oxydation le plus avancé, on parle de mélange stœchiométrique (par exemple $\mathrm{CH}_{4}+2 \mathrm{O}_{2}=\mathrm{CO}_{2}+2 \mathrm{H}_{2} \mathrm{O}$ ). La richesse (équivalence ratio) désigne le rapport adimensionnel de la quantité relative de combustible à cette même quantité relative dans le mélange stœchiométrique (par définition de richesse 1).

Les processus de combustion sont l'objet de couplages complexes entre mécanique des fluides, transport, cinétique chimique. Afin de comprendre les mécanismes chimiques détaillés qui permettent 


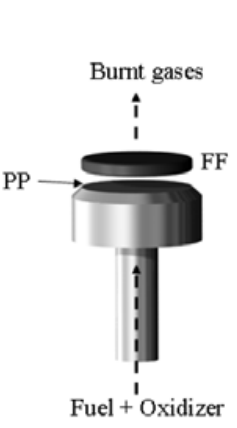

a)

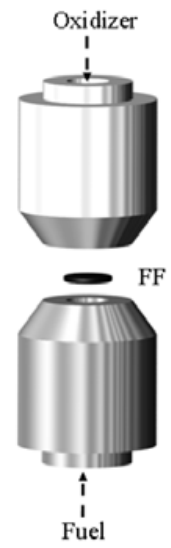

b)

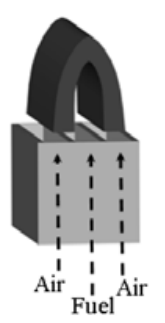

c)

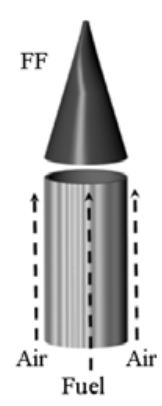

d)

Figure 1. Illustration de : (a) flamme plate stabilisée sur poreux (b) flamme non prémélangée à contre-courant, (c) flamme non prémélangée (de diffusion) stabilisée sur un brûleur à trois fentes (Wolfhard-Parker burner) et (d) flamme de diffusion coaxiale. PP : poreux, FF : front de flamme.

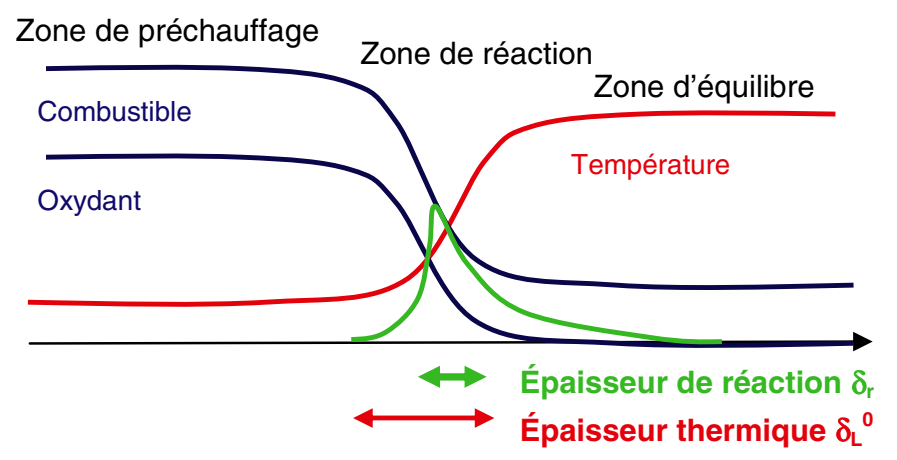

Figure 2. Représentation schématique d'une flamme de prémélange.

de rendre compte de l'oxydation thermique d'un combustible donné, on a en général recours à des systèmes simplifiés dans lesquels les phénomènes de transport sont facilement modélisables : flammes plates, réacteurs auto-agités, tubes à choc.

Les flammes plates sont stabilisées sur des poreux traversés par un prémélange combustible/ comburant. Elles sont unidimentionnelles, c'est-à-dire que les champs de concentration des espèces et de la température sont homogènes dans des plans parallèles à la surface du brûleur. Le traitement théorique des flammes monodimensionnelles et stationnaires a été réalisé par Zeldovich et Frank-Kamenetzki (1938). La zone de combustion peut être divisée en trois parties : la zone de préchauffage, la zone de réaction et la zone d'équilibre. On peut suivre le processus de combustion en étudiant l'évolution des concentrations d'espèces (u.a.) à différentes hauteurs au dessus du brûleur (HAB). La figure 3 illustre un exemple de profils de quelques concentrations d'espèces et de particules de suies obtenues dans une flamme suitée de prémélange méthane/oxygène/azote.

L'établissement de bases de données obtenues dans des conditions opératoires couvrant un large domaine de température et de pression permet de valider les mécanismes chimiques détaillés de combustion. Un exemple de mécanisme détaillé (partiel) rendant compte des réactions de consommation du benzène est donné figure 4. L'utilisation de codes de simulation de flammes (le plus utilisé est 
Chemistry in Astrophysical Media, AstrOHP 2010

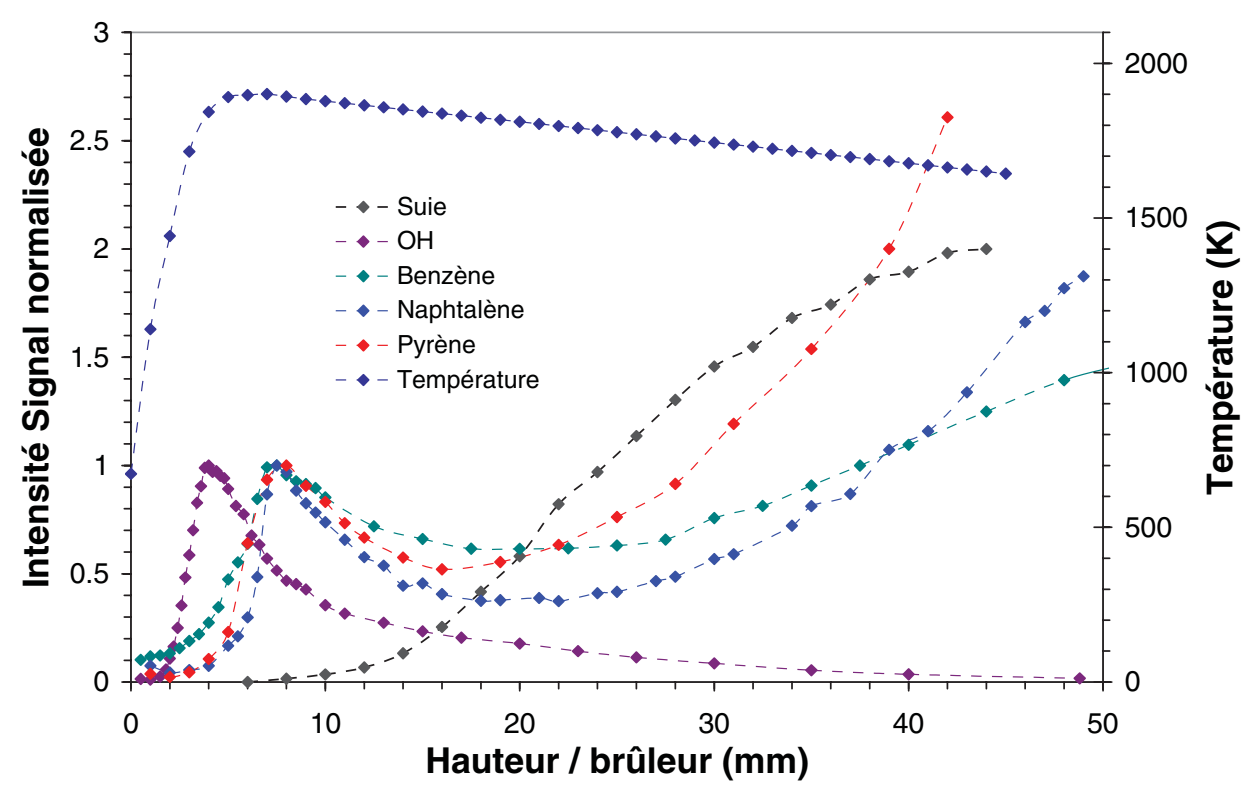

Figure 3. Exemple de structure chimique d'une flamme prémélangée suitée. Thèse M. Wartel (en cours, PC2A, Univ. Lille 1).

\begin{tabular}{|c|c|c|c|}
\hline Reaction & A & $\mathrm{n}$ & $\mathrm{E}_{a}$ [cal] \\
\hline Benzene $+\mathrm{OH} \leftrightharpoons$ phenyl $+\mathrm{H}_{2} \mathrm{O}$ & $2.11 \times 10^{13}$ & 0.0 & 4571 \\
\hline Benzene $+\mathrm{O} \leftrightharpoons$ phenoxy $+\mathrm{H}$ & $2.40 \times 10^{13}$ & 0.0 & 4668 \\
\hline Benzene $+\mathrm{H} \leftrightharpoons$ phenyl $+\mathrm{H}_{2}$ & $3.23 \times 10^{7}$ & 2.095 & 15842 \\
\hline Phenyl $+\mathrm{H} \leftrightharpoons$ benzene & $8.02 \times 10^{19}$ & -2.011 & 1968 \\
\hline Benzene $+\mathrm{OH} \leftrightharpoons$ phenol $+\mathrm{H}$ & $1.59 \times 10^{19}$ & -1.82 & 12800 \\
\hline Phenyl $+\mathrm{O}_{2} \leftrightharpoons$ phenoxy $+\mathrm{O}$ & $2.39 \times 10^{21}$ & -2.62 & 4400 \\
\hline Phenyl $+\mathrm{O}_{2} \leftrightharpoons$ o-benzoquinone $+\mathrm{H}$ & $3.00 \times 10^{13}$ & 0.0 & 9000 \\
\hline Phenyl $+\mathrm{OH} \leftrightharpoons$ phenoxy $+\mathrm{H}$ & $5.00 \times 10^{13}$ & 0.0 & 0 \\
\hline Phenyl $+\mathrm{O} \leftrightharpoons \mathrm{c}-\mathrm{C}_{5} \mathrm{H}_{5}+\mathrm{CO}$ & $9.00 \times 10^{13}$ & 0.0 & 0 \\
\hline $\mathrm{i}-\mathrm{C}_{4} \mathrm{H}_{3}+\mathrm{C}_{2} \mathrm{H}_{3} \leftrightharpoons$ phenyl $+\mathrm{H}$ & $6.00 \times 10^{12}$ & 0.0 & 0 \\
\hline Phenoxy $+\mathrm{H} \leftrightharpoons$ phenol & $4.43 \times 10^{60}$ & -13.232 & 30010 \\
\hline Phenol $+\mathrm{OH} \leftrightharpoons$ phenoxy $+\mathrm{H}_{2} \mathrm{O}$ & $1.39 \times 10^{8}$ & 1.43 & -962 \\
\hline Phenol $+\mathrm{H} \leftrightharpoons$ phenoxy $+\mathrm{H}_{2}$ & $1.15 \times 10^{14}$ & 0.0 & 12400 \\
\hline Phenol $\leftrightharpoons \mathrm{c}-\mathrm{C}_{5} \mathrm{H}_{6}+\mathrm{CO}$ & $1.00 \times 10^{12}$ & 0.0 & 60802 \\
\hline Phenoxy $\leftrightharpoons \mathrm{c}-\mathrm{C}_{5} \mathrm{H}_{5}+\mathrm{CO}$ & $2.51 \times 10^{11}$ & 0.0 & 43900 \\
\hline $\mathrm{c}-\mathrm{C}_{5} \mathrm{H}_{5}+\mathrm{H} \leftrightharpoons \mathrm{c}-\mathrm{C}_{5} \mathrm{H}_{6}$ & $2.71 \times 10^{63}$ & -14.79 & 21050 \\
\hline$c-\mathrm{C}_{5} \mathrm{H}_{6}+\mathrm{H} \leftrightarrows \mathrm{c}-\mathrm{C}_{5} \mathrm{H}_{5}+\mathrm{H}_{2}$ & $2.80 \times 10^{13}$ & 0.0 & 2259 \\
\hline $\mathrm{c}-\mathrm{C}_{5} \mathrm{H}_{5}+\mathrm{O} \leftrightharpoons \mathrm{c}-\mathrm{C}_{5} \mathrm{H}_{5}+\mathrm{OH}$ & $4.77 \times 10^{4}$ & 2.71 & 1106 \\
\hline $\mathrm{c}-\mathrm{C}_{5} \mathrm{H}_{6}+\mathrm{OH} \leftrightharpoons \mathrm{c}-\mathrm{C}_{5} \mathrm{H}_{5}+\mathrm{H}_{2} \mathrm{O}$ & $3.08 \times 10^{6}$ & 2.00 & 0 \\
\hline $\mathrm{c}-\mathrm{C}_{5} \mathrm{H}_{5}+\mathrm{O}=\mathrm{c}-\mathrm{C}_{5} \mathrm{H}_{4} \mathrm{O}+\mathrm{H}$ & $6.71 \times 10^{13}$ & -0.03 & 40 \\
\hline $\mathrm{c}-\mathrm{C}_{5} \mathrm{H}_{4} \mathrm{O}+\mathrm{H} \leftrightharpoons \mathrm{n}-\mathrm{C}_{4} \mathrm{H}_{5}+\mathrm{CO}$ & $2.10 \times 10^{61}$ & -13.27 & 40810 \\
\hline $\mathrm{c}-\mathrm{C}_{5} \mathrm{H}_{5}+\mathrm{OH} \leftrightharpoons \mathrm{c}-\mathrm{C}_{5} \mathrm{H}_{4}+\mathrm{H}_{2} \mathrm{O}$ & $2.11 \times 10^{13}$ & 0.0 & 4571 \\
\hline $\mathrm{c}-\mathrm{C}_{5} \mathrm{H}_{5}+\mathrm{H} \leftrightharpoons \mathrm{c}-\mathrm{C}_{5} \mathrm{H}_{4}+\mathrm{H}_{2}$ & $3.23 \times 10^{7}$ & 2.095 & 15842 \\
\hline $\mathrm{c}-\mathrm{C}_{5} \mathrm{H}_{5}+\mathrm{O} \leftrightharpoons \mathrm{n}-\mathrm{C}_{4} \mathrm{H}_{5}+\mathrm{CO}$ & $7.27 \times 10^{13}$ & -0.28 & 470 \\
\hline $\mathrm{c}-\mathrm{C}_{5} \mathrm{H}_{6}+\mathrm{H} \leftrightharpoons \mathrm{C}_{3} \mathrm{H}_{5}+\mathrm{C}_{2} \mathrm{H}_{2}$ & $6.60 \times 10^{14}$ & 0.0 & 12345 \\
\hline $0-\mathrm{C}_{6} \mathrm{H}_{4} \mathrm{O}_{2}=\mathrm{c}-\mathrm{C}_{5} \mathrm{H}_{4} \mathrm{O}+\mathrm{CO}$ & $1.00 \times 10^{12}$ & 0.0 & 40000 \\
\hline
\end{tabular}

Figure 4. Exemple de mécanisme (partiel) de la consommation du benzène [2]. 


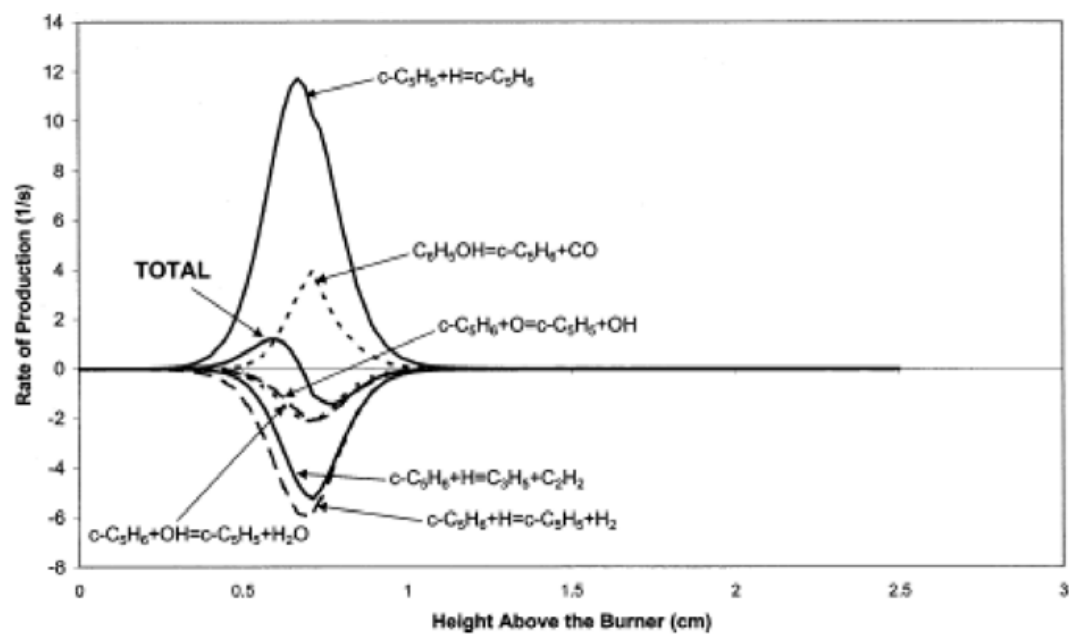

Figure 5. Analyse des vitesses de production du cyclopentadiène impliqué dans le mécanisme de consommation du benzène [2].

PREMIX/CHEMKIN [1] pour les flammes de prémélange) permet de résoudre le système d'équations différentielles représentatives des lois de conservation de la masse totale, des espèces et de l'énergie (équations de Navier-Stokes). Le mécanisme chimique est optimisé par rapport à la base de données expérimentales. Lorsque un accord satisfaisant est obtenu, on peut réaliser une analyse des vitesses de production mises en jeu ce qui permet par exemple de déceler quelles sont les réactions importantes intervenant lors de la consommation ou de la production d'une espèce particulière (le cyclopentadiène c- $\mathrm{C}_{5} \mathrm{H}_{6}$ dans le cas de la figure 5).

\section{LA FORMATION DES SUIES DANS LES FLAMMES}

Lors d'une combustion, le carbone du combustible est transformé par une succession de réactions chimiques en $\mathrm{CO}_{2}$ et en $\mathrm{CO}$. Si cette combustion se déroule en défaut important de dioxygène (c'està-dire dans le cas d'une combustion dite très riche), des particules carbonées solides appelées suies apparaissent dans la zone des gaz brûlés. Le passage d'un système gazeux composé du combustible, de l'oxydant et de l'ensemble d'intermédiaires chimiques stables ou radicalaires à un système comportant des particules solides dans un environnement de gaz brûlés est encore aujourd'hui un problème complexe non résolu de la combustion. Même si l'on connait les grandes lignes qui permettent d'expliquer la formation des particules de suie, l'ultime étape conduisant à la première particule (primary particle, nascent particle) n'est pas connue. Avant de décrire plus en détail ces différentes étapes, une brève description des particules de suie est présentée.

\subsection{Description des particules de suies}

On s'intéresse ici uniquement à la morphologie et la composition chimique des suies. Il existe bien d'autres caractéristiques notamment les propriétés optiques nécessaires pour les diagnostics optiques de suies (cf. chapitre 4). Les particules de suies peuvent avoir des aspects morphologiques très différents. La figure 6 représente deux clichés obtenus par microscopie TEM sur des échantillons de suie prélevés dans une même flamme sur une grille TEM (lacey carbon) par effet thermophorétique. Sur le cliché gauche, obtenu haut dans la flamme (c'est-à-dire après un long temps de résidence), les suies se présentent sous forme d'agrégats. 

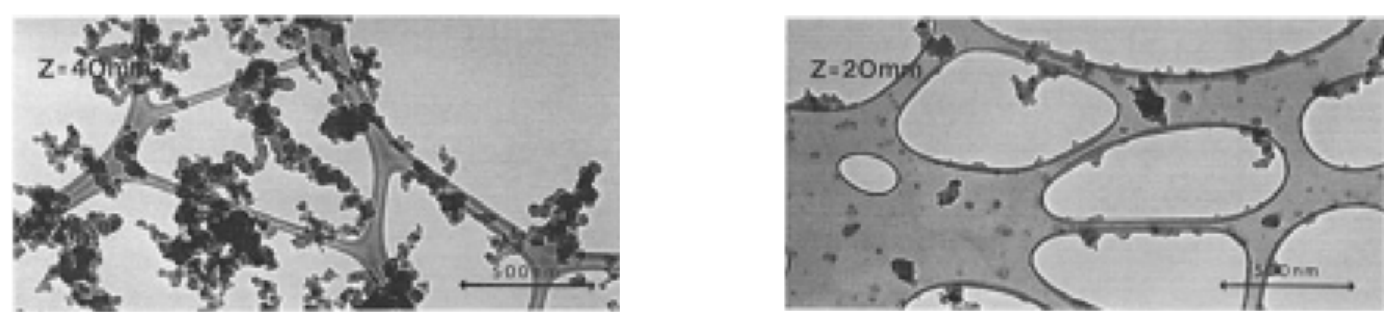

Figure 6. Particules capturées sur des grilles TEM (lacey carbon) dans une flamme d'éthylène : agrégats (gauche) et nanoparticules (PNP) (à droite). D’après Dobbins [3].

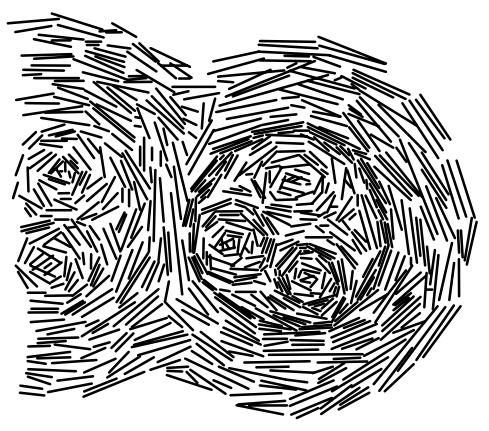

Figure 7. Structure d'une suie «Diesel». D'après Ishiguro [4].

On y distingue nettement les particules primaires sphériques qui les constituent. Leur structure est bien connue. Elle est composée d'un empilement de cristallites orientées de manière parallèle dans la couronne externe de la suie et aléatoirement en son cœur (figure 7).

Le nombre de particules par agrégat, la structure tridimensionnelle de l'agrégat (souvent représentée par sa dimension fractale) dépendent de la zone de la flamme, de l'avancement de la réaction, du combustible etc. Sur le cliché 6 (droite), obtenu plus bas dans la flamme (c'est-à-dire pour un temps de séjour court), le dépôt a plutôt l'aspect de gouttes. Les «particules» apparaissent collées sur les bords de la grille par des forces de tension superficielles. Ces tensions superficielles sont interprétées comme provenant d'un état liquide de la matière d'où l'appellation liquid-like particles par certains auteurs. Dobbins [5] les appelle precursor nanoparticle (PNP) alors que d'autres équipes les nomment Nanoparticles Organic Carbon (NOC) [6]. Ces entités sont peut-être le signe de l'existence d'un état intermédiaire au sein de la flamme entre la phase gaz et l'apparition des premières particules primaires solides. Ces observations sont cohérentes avec les distributions de taille de particules obtenues après prélèvement et dilution par la technique Scanning Mobility Particle Sizing (SMPS) qui mettent en exergue une distribution bimodale des particules (figure 8) avec des PNP de taille nanométrique et des particules de suies dont le diamètre de mobilité se situe dans une fourchette $10-50 \mathrm{~nm}$.

Les particules de suies prélevées à un stade avancé de la combustion dans la flamme sont essentiellement composées de carbone et pour quelques \% de leur masse par des atomes d'hydrogène. La connaissance des espèces chimiques adsorbées à la surface des particules est utile pour comprendre les processus de croissance des suies dans la flamme. La figure 9 représente un spectre de masse caractéristique de la composition de surface d'une suie. Il s'avère que tous les pics observés correspondent à des hydrocarbures aromatiques polycycliques (HAP), dont la structure correspond à celle calculée par Stein and Fahr sur des bases thermodynamiques [8]. A partir de l'analyse des spectres de masse on peut établir des diagrammes H-C tout le long de la flamme. On peut constater que le rapport $\mathrm{C} / \mathrm{H}$ augmente avec le temps de séjour de la flamme ce qui traduit une «maturation» des particules de 

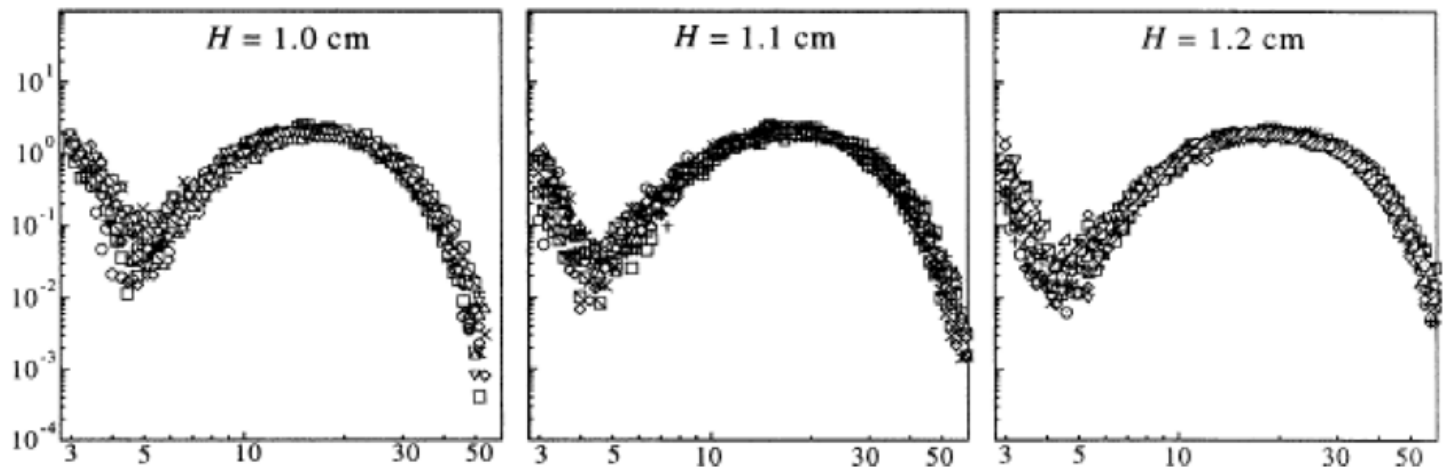

Figure 8. Distribution bimodale (pdf normalisée en fonction du diamètre de la particule primaire (nm)) de tailles de particules à différnets positions dans une flamme d'éthylène. D'après Zhao [7].
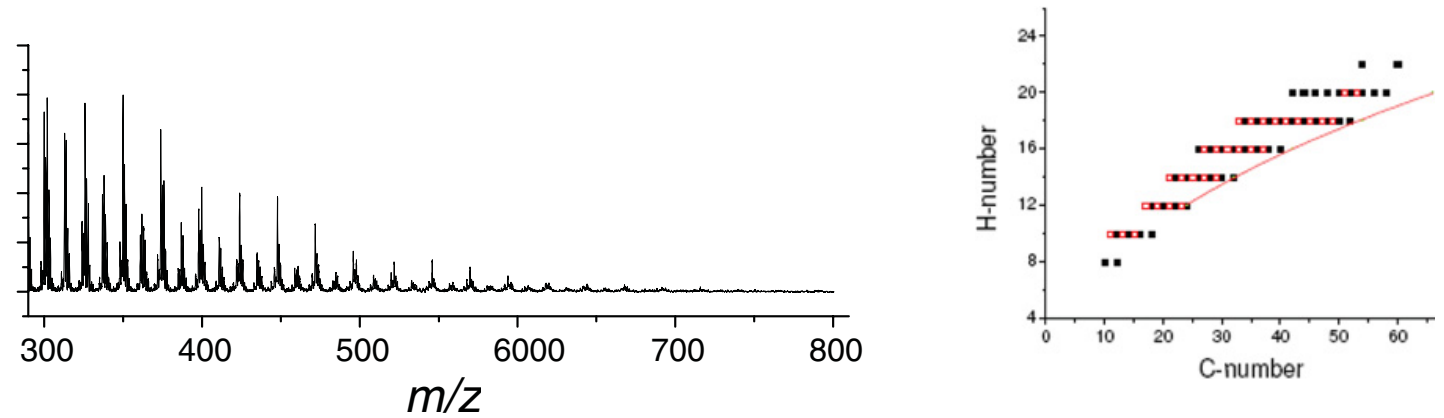

Figure 9. Spectre de masse de suies prélevées dans une flamme $\mathrm{C}_{2} \mathrm{H}_{4}$ /Air obtenu par désorption laser/ionisation laser/spectrométrie de masse à temps de vol et l'analyse en masse représentée au moyen d'un diagramme H-C. Thèse A. Faccinetto, Univ. Lille 1 (2009) [10].

suie dont la structure tend à se rapprocher de celle du graphite. (Il est à noter que la composition chimique des suies collectées dans l'atmosphère présente d'autres groupements, notamment oxygénés du fait des réactions hétérogènes qu'elles subissent après un long temps de séjour dans l'atmosphère.)

Certains auteurs préconisent de «déshabiller» les suies à différentes HAB pour identifier les espèces gazeuses environnantes qui participent au processus de croissance de surface des suies [9].

\subsection{Les étapes conduisant aux particules de suie dans les flammes}

De très bonnes revues font un point détaillé sur ces étapes. Citons Richter [11] et Frenklach [12]. La figure 10, extraite de Bockhorn [13] décrit très bien la succession des étapes donnant naissance aux particules.

Depuis deux décennies, un consensus est établi, sur la base d'observations expérimentales, pour désigner les HAP de poids moléculaire élévé comme étant les espèces moléculaires précurseurs des suies les plus probables. Notons que ceci n'exclut pas l'existence d'une entité intermédiaire entre les HAP précurseurs et les particules primaires. 


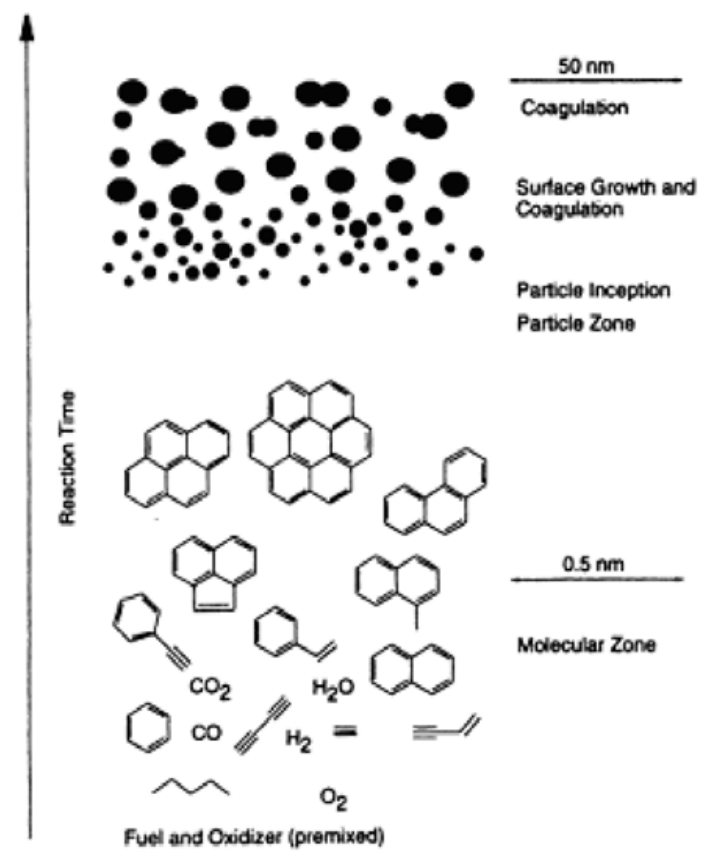

Figure 10. Étapes de formation des particules de suies, d'après Bockhorn [13].

à

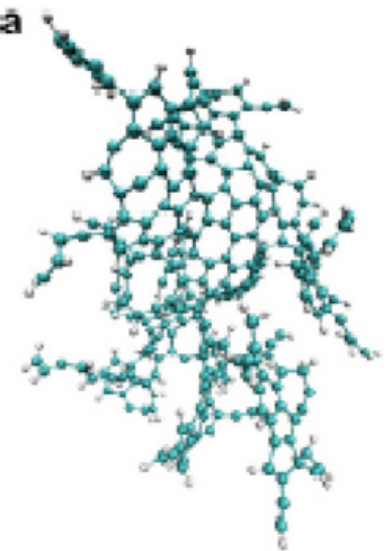

b

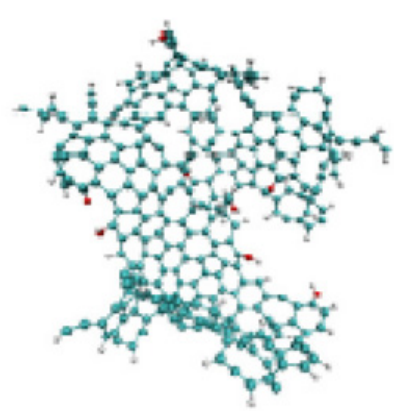

c

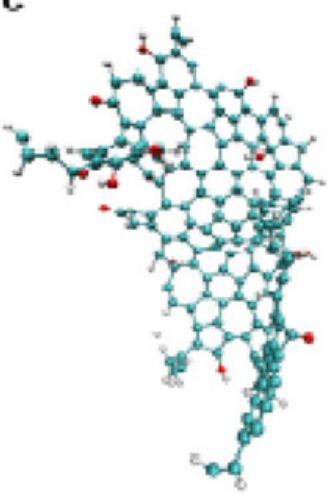

Figure 11. Carbonaceous nanoparticles calculées à partir du code AMPI (Atomistic Model for Particle Inception). Bleu $(\mathrm{C})$, rouge $(\mathrm{O})$, argent $(\mathrm{H})$. D'après Chung and Violi [15].

- La nucléation.

Au cours de la nucléation, on passe d'un système moléculaire à un système particulaire ; c'est-à-dire que des HAP de poids moléculaire élevé (gazeux) forment les premières particules (nascent particles). Ce processus n'est pas encore compris. Certains proposent l'existence de clusters tridimensionnels (three-dimensional layered oligomers) formés à partir de la coalescence de HAP [14]. Ce processus l'emporterait sur le processus de croissance de HAP sous forme d'oligomères plans. Ces structures complexes peuvent être calculées au moyen de la dynamique moléculaire [15].

Compte-tenu de ces avancées, D’Anna propose le schéma suivant [16] : 


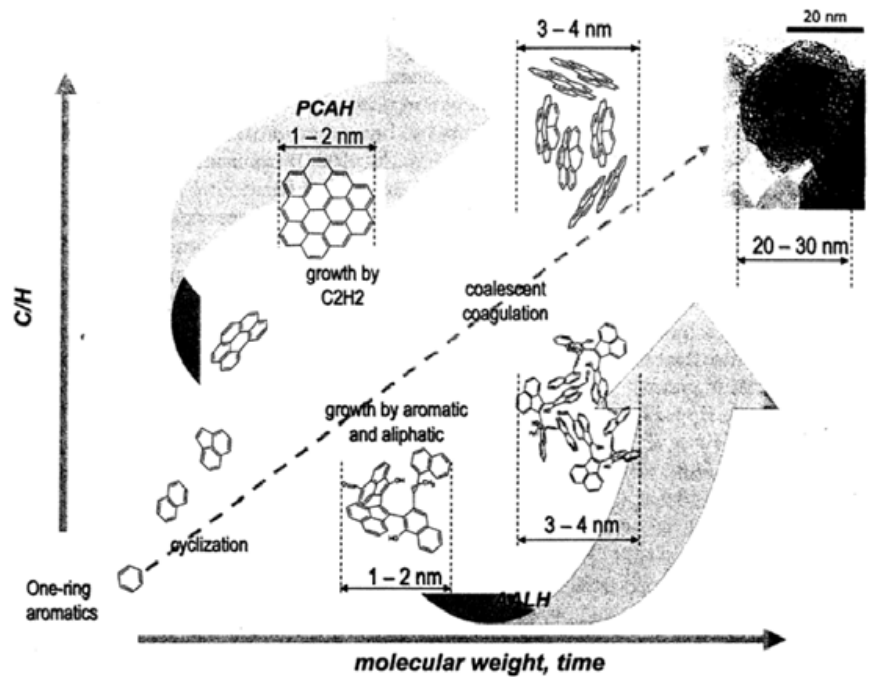

Figure 12. Diagramme illustrant les voies possibles de formation des suies d'après D'Anna [16]. Par addition successive de $\mathrm{C}_{2} \mathrm{H}_{2}$ selon un mécanisme de type HACA, la croissance des aromatiques conduit à de larges aromatiques péricondensés (peri-condensed aromatic hydrocarbons (PCAH)). Au contraire, l'addition de molécules aromatiques à des radicaux aromatiques conduit à des structures tridimensionnelles liées éventuellement par des chaines aliphatiques, avec l'inclusion possible d'atomes d'oxygène (aromatic-aliphatic-linked hydrocarbons $(\mathrm{AALH})$ ). Ce type de réaction est favorable à la formation de HAP contenant 5 membres.

Les processus physicochimiques qui succèdent à la nucléation sont davantage compris. Néanmoins les valeurs quantitatives de l'ensemble des coefficients qui interviennent pour décrire la coagulation, l'agrégation ou les réactions de surface sont affectées d'une très large incertitude.

- La croissance de surface.

Après la nucléation de petites particules, leur augmentation de masse s'effectue par des réactions de croissance de surface mettant en jeu un site disponible de la particule et une espèce gazeuse de la flamme. Il s'agit essentiellement de l'acétylène $\mathrm{C}_{2} \mathrm{H}_{2}$ et de HAP stables ou radicalaires. La croissance de surface est le processus qui est responsable de la majeure partie de l'augmentation de la masse de suies dans les flammes de prémélange. Ces réactions suivent une cinétique de $1^{\text {er }}$ ordre selon la loi de vitesse suivante :

$$
\frac{d f_{v}}{d t}=k_{f}\left(f_{v \infty}-f_{v}\right)
$$

Où $f_{v \infty}$ désigne la fraction volumique finale de suie, $f_{v}$ la fraction volumique de suie et $k_{f}$ la constante de croissance empirique $\left(\mathrm{s}^{-1}\right)$.

La plupart des modèles ne considèrent qu'une croissance par addition d'acétylène (rarement par condensation de $\mathrm{HAP}$ ) et une consommation par réaction avec $\mathrm{O}_{2}$ et le radical $\mathrm{OH}$ :

$$
\begin{aligned}
& \mathrm{C}_{\text {soot }}-\mathrm{H}+\mathrm{H} \leftrightarrow \mathrm{C}_{\text {soot }}^{\bullet}+\mathrm{H}_{2} \\
& \mathrm{C}_{\text {soot }}^{\bullet}+\mathrm{H} \rightarrow \mathrm{C}_{\text {soot }}-\mathrm{H} \\
& \mathrm{C}_{\text {soot }}^{\bullet}+\mathrm{C}_{2} \mathrm{H}_{2} \rightarrow \mathrm{C}_{\text {soot }}-\mathrm{H}+\mathrm{H} \\
& \mathrm{C}_{\text {soot }}^{\circ}+\mathrm{O}_{2} \rightarrow \text { produits }
\end{aligned}
$$

Csoot ${ }^{\bullet}$ désigne le radical produit par Csoot - $\mathrm{H}$ suite à un transfert de l'atome d'hydrogène par $\mathrm{H}$.

- La coagulation.

La description mathématique du processus de coagulation des particules est réalisée en considérant un ensemble homogène de particules (aérosol) dans l'espace et dont le volume est supposé être la somme 
des volumes des particules primaires. En supposant que la densité des particules est indépendante de la dimension particulaire, on obtient une relation semblable d'additivité pour la masse $\mathrm{m}_{i}$ des particules. La coagulation de particules par collision contribue grandement à l'augmentation de leur taille tout en diminuant leur nombre.

- L'agrégation.

Dans de nombreux cas, après une première période de croissance par coalescence, des particules commencent à s'agglomérer. Les agglomérats obtenus prennent habituellement la forme de structures 3D composées de particules primaires quasi sphériques et dont l'agencement peut être représenté par la relation suivante :

$$
n=k_{\mathrm{f}}\left(\frac{2 R_{\mathrm{g}}}{d_{\mathrm{p}}}\right)^{D f}
$$

$\mathrm{n}$ désigne le nombre de particules primaires dans un agrégat, $\mathrm{d}_{\mathrm{p}}$ le diamètre des particules primaires, $\mathrm{R}_{\mathrm{g}}$ le rayon de giration d'un agrégat, $\mathrm{D}_{\mathrm{f}}$ est la dimension fractale et $\mathrm{k}_{f}$ est appelé le pré-facteur fractal.

- Carbonisation/oxydation.

Selon l'environnement gazeux de la particule lors de son temps de séjour dans la flamme, celle-ci pourra soit tendre vers une carbonisation en l'absence d'oxygène (c'est-à-dire que l'on se retrouve dans des conditions de pyrolyse, cas des flammes de prémélange), ou vers une oxydation : la suie disparaît au profit du $\mathrm{CO}\left(\right.$ ou $\mathrm{CO}_{2}$ ). L'oxydation se manifeste par exemple dans les flammes de diffusion suitées mais non fumantes (sooting but not smoking).

\subsection{Les modèles de formation des suies}

Des lois empiriques ou semi-empiriques établissent des corrélations entre une concentration de suies et certains paramètres initiaux. Ils sont très utiles pour estimer la teneur en suies dans un cadre opératoire très restreint (limité par exemple à des expériences en tube à choc) [17]. Pour la compréhension et la validation des lois physico-chimiques introduites, des modèles détaillés ont été développés. On distingue essentiellement deux approches mathématiques: la méthode des moments et la méthode par sections discrètes.

Les modèles s'appuient sur l'équation de Smoluchowsky qui décrit la cinétique de formation de particules de type (i) (particules ayant une masse $\mathrm{mi} \pm \delta \mathrm{m}$ par exemple) par un nombre infini d'équations différentielles du type :

$$
\begin{aligned}
\frac{\mathrm{d} N_{t}}{\mathrm{~d} t} & =\frac{1}{2} \sum_{j=1}^{i-1} \beta_{j, i-j} N_{j} N_{i-j}-\sum_{j=1}^{\infty} \beta_{i, j} N_{i} N_{j}, \\
i & =2, \ldots, \infty .
\end{aligned}
$$

où les 2 termes à droite de l'égalité désignent respectivement les taux d'apparition et de disparition de l'entité (i) par collisions avec ses partenaires de collisions (j), $\beta_{i j}$ désignant la fréquence de collision entre l'espèce (i) et l'espèce $(\mathrm{j})$ et $\mathrm{N}_{i}$ la densité de l'espèce de type (i). La «collision» peut désigner l'ensemble des processus intervenant dans la formation des suies : nucléation, croissance de surface, coagulation et agrégation. Dans l'équation ci-dessus, la nature de la collision se traduit par un choix donné des termes $\beta_{i j}$ (on renvoie le lecteur aux références $\left.[18,19]\right)$ pour de plus amples explications). Cette description s'appuie sur la physique de la dynamique des aérosols (de type suie). Par exemple le terme de coagulation est fortement dépendant du régime de coagulation dans lequel se situe le système (régime continu, régime moléculaire libre ou régime transitoire) déterminé par la valeur du nombre de Knudsen.

La nucléation est modélisée par une réaction de coagulation entre 2 HAP pour former un dimère (le dimère devient donc le $1^{\mathrm{er}}$ nucléus). Si toutes les réactions de coalescence peuvent en principe être introduites dans le modèle, on se limite généralement à la coalescence de deux pyrènes (HAP à 4 cycles 


\section{EPJ Web of Conferences}

$\mathrm{C}_{20} \mathrm{H}_{12}$ ) pour rendre compte de la nucléation, faute de prédiction suffisamment précise pour les HAP plus lourds. Récemment, Sabbah et al. [20] ont prouvé que la dimérisation du pyrène ne pouvait se produire en condition de flamme. Cette avancée doit inciter les chercheurs à la prise en considération d'autres voies impliquant des HAP de taille plus importante.

La méthode des moments est une méthode statistique. Le moment d'ordre $\mathrm{r} \mathrm{M}_{r}$ est défini comme la somme d'une grandeur $x_{i}^{r}$ pondérée par une fonction de probabilité $\mathrm{p}_{i}$. Si l'on décompose les particules suies selon leur distribution en masse, on peut définir le moment d'ordre r par :

$$
M_{r}=\sum_{i=1}^{\infty} i^{r} N_{i}
$$

où $\mathrm{N}_{\mathrm{i}}$ représente la concentration des particules de masse $\mathrm{m}_{\mathrm{i}}=\mathrm{im}_{1}, \mathrm{~m}_{1}$ étant la masse de la plus petite particule de suie.

Le premier moment $\mathrm{M}_{0}$ correspond à la concentration totale de particules et le moment $\mathrm{M}_{1}$ :

$$
\begin{gathered}
M_{1}=\sum_{i=1}^{\infty} i N_{i}=f_{v} \frac{\rho_{s}}{m_{1}} \text { permet de calculer la fraction volumique } \mathrm{f}_{\mathrm{v}} \text { des suies } \\
\text { ( } \left.\rho_{\mathrm{s}} \text { étant la masse volumique des suies }\right) .
\end{gathered}
$$

On peut monter que le système d'équations différentielles (1) peut se réécrire sous la forme d'une succession d'équations représentatives des moments d'ordre (r) définis par :

$$
\begin{gathered}
\frac{d M_{0}}{d t}=-\frac{1}{2} \sum_{i, j=1}^{\infty} \beta_{i, j} N_{i} N_{j} \\
\frac{d M_{1}}{d t}=0 \quad \text { (conservation de la masse) } \\
\frac{d M_{2}}{d t}=\sum_{i=1}^{\infty} \sum_{i=1}^{\infty} i j \beta_{i, j} N_{i} N_{j} \\
\frac{d M_{3}}{d t}=3 \sum_{i=1}^{\infty} \sum_{i=1}^{\infty} i j^{2} \beta_{i, j} N_{i} N_{j}
\end{gathered}
$$

etc.

En introduisant les termes $\mathrm{R}, \mathrm{G}$, et $\mathrm{W}$ désignant respectivement la nucléation, la coagulation et la croissance de surface, on obtient :

$$
\begin{gathered}
\frac{d M_{0}}{d t}=R_{0}-G_{0} \\
\frac{d M_{1}}{d t}=R_{1}+W_{0} \\
\frac{d M_{2}}{d t}=R_{2}+G_{2}+W_{2} \\
\frac{d M_{r}}{d t}=R_{r}+G_{r}+W_{r} .
\end{gathered}
$$

La connaissance de l'ensemble des moments permet une description complète du système. En pratique on s'intéresse surtout aux deux premiers moments. Ainsi la méthode des moments permet de remplacer 
un nombre infini d'équations différentielles en un nombre limité d'équations donnant une bonne description du système. Une difficulté de cette méthode réside dans le choix des équations de fermeture. On impose en général une loi de distribution de taille (particule size distribution function) à ces fins.

Une approche différente a été proposée par Pope and Howard [21], afin de décrire la dynamique de l'aérosol de particules sous une forme compatible pour être combinée aisément au modèle cinétique détaillé décrivant la phase gazeuse. Dans cette approche par sections discrètes (discrete sectional technique), l'aérosol de particules est traité comme un type de molécules dont la cinétique peut être représentée par des réactions élémentaires incorporables dans le modèle détaillé décrivant la phase gazeuse. Les particules sont regroupées par classes de taille, chaque classe étant caractérisée par une particule «moyenne» affectée d'une masse moyenne et leur cinétique est représentée sous la forme d'une loi d'Arrhénius. Des détails sur cette méthode sont disponibles dans les références [22, 23].

\section{FORMATION DES HYDROCARBURES AROMATIQUES POLYCYCLIQUES}

Dans cette partie les principales voies de formation de HAP sont décrites. Ce sujet de recherche est toujours en plein essor.

\subsection{Formation du premier noyau aromatique}

En se basant sur une étude par modélisation de la pyrolyse de l'acétylène en tube à choc, une voie réactionnelle $\mathrm{C}_{4} / \mathrm{C}_{2}$ a été suggérée, pour expliquer la formation du premier noyau aromatique [12]. Deux réactions impliquant l'acétylène ont été proposées :

$$
\begin{gathered}
\text { n- } \mathrm{C}_{4} \mathrm{H}_{3}+\mathrm{C}_{2} \mathrm{H}_{2} \rightarrow \text { phényle } \\
\text { n- } \mathrm{C}_{4} \mathrm{H}_{5}+\mathrm{C}_{2} \mathrm{H}_{2} \rightarrow \text { benzène }+\mathrm{H}
\end{gathered}
$$

Les réactions (1) et (2) sont, selon Miller et Melius, insuffisantes et ne peuvent expliquer, à elles seules, la formation du noyau aromatique en raison de la faible concentration des radicaux $n-\mathrm{C}_{4} \mathrm{H}_{3}$ et $n-\mathrm{C}_{4} \mathrm{H}_{5}$ [24]. Ces auteurs ont alors suggéré une nouvelle de voie de formation du cycle aromatique qui implique la recombinaison des radicaux propargyle :

$$
\mathrm{C}_{3} \mathrm{H}_{3}+\mathrm{C}_{3} \mathrm{H}_{3} \rightarrow \text { [phényle }+\mathrm{H} \text { ] ou benzène }
$$

Certains auteurs, en particulier Marinov [25], attribuent un rôle central au cyclopentadiène dans la formation du benzène. Le radical cyclopentadiényle peut soit se combiner avec lui même ou avec le radical méthyle pour former respectivement le naphtalène et le benzène :

$$
\begin{gathered}
\mathrm{C}_{5} \mathrm{H}_{5}+\mathrm{CH}_{3} \rightarrow \text { benzène }+\mathrm{H}+\mathrm{H} \\
\mathrm{C}_{5} \mathrm{H}_{5}+\mathrm{C}_{5} \mathrm{H}_{5} \rightarrow \text { Naphtalène }+\mathrm{H}+\mathrm{H}
\end{gathered}
$$

Le radical cyclopentadiénlyle $\mathrm{C}_{5} \mathrm{H}_{5}$ est produit à partir des radicaux propargyle et l'acétylène :

$$
\mathrm{C}_{3} \mathrm{H}_{3}+\mathrm{C}_{2} \mathrm{H}_{2} \rightarrow \mathrm{C}_{5} \mathrm{H}_{5}
$$

Cette dernière réaction est favorisée en milieu très riche, en raison de l'abondance de l'acétylène d'une part, et de la grande stabilité des radicaux propargyle d'autre part.

\subsection{Formation des premiers HAP (HAP de petite taille)}

Le mécanisme HACA «H-Abstraction- $\mathrm{C}_{2} \mathrm{H}_{2}$-Addition» proposé par Frenklach et Wang [26] est le plus admis actuellement par la communauté scientifique. Il s'inspire des travaux de Bittner et Howard [27]. Le mécanisme HACA considère une succession de deux réactions répétitives : activation par abstraction 
DOMINANT ROUTE

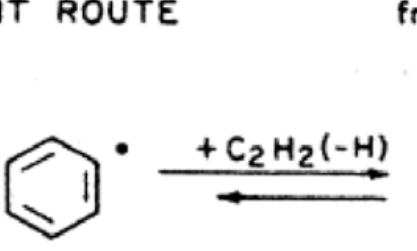

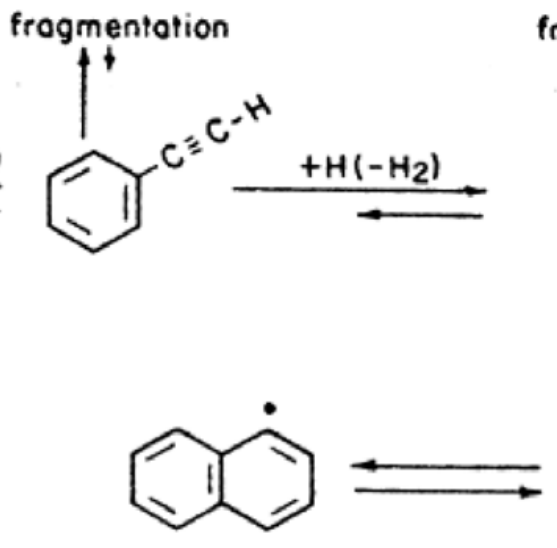<smiles>CC#Cc1ccccc1C#CCCCCCO</smiles><smiles>CC#CC=Cc1ccccc1C=CC</smiles>

Figure 13. Voie dominante de formation d'un HAP à 2 cycles selon le mécanisme HACA (d'après Richter [11]).

d'un atome d'hydrogène d'une molécule $A_{i}$ par un atome d'hydrogène présent dans la phase gaz suivi d'une addition d'une molécule d'acétylène sur le site radicalaire formé :

$$
\begin{gathered}
\mathrm{A}_{\mathrm{i}}+\mathrm{H} \rightarrow \mathrm{A}_{\mathrm{i}-}+\mathrm{H}_{2} \\
\mathrm{~A}_{\mathrm{i}-}+\mathrm{C}_{2} \mathrm{H}_{2} \rightarrow \text { produits }
\end{gathered}
$$

$\mathrm{A}_{i}$ est une molécule aromatique et i désigne le nombre de noyau aromatique, $\mathrm{A}_{i-}$ est le radical qui en est issu par élimination d'un atome d'hydrogène. Le processus d'abstraction est réversible, en d'autres termes, le composé radicalaire peut se désactiver en réagissant avec l'hydrogène moléculaire ou l'atome $\mathrm{H}$ pour redonner la molécule de départ.

$$
\mathrm{A}_{\mathrm{i}-}+\mathrm{H}_{2} \rightarrow \mathrm{A}_{\mathrm{i}}+\mathrm{H}
$$

$$
\mathrm{A}_{\mathrm{i}-}+\mathrm{H} \rightarrow \mathrm{A}_{\mathrm{i}}
$$

Le degré de réversibilité de l'étape d'addition d'acétylène détermine si cette étape contribuera de façon significative à la croissance des HAP.

Il est également possible de produire le biphényle plutôt que le naphtalène par addition du benzène sur le radical phényle :

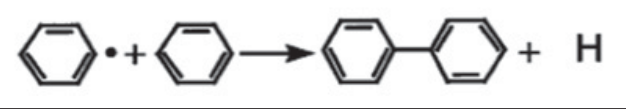

Ce processus est ensuite suivi par l'addition de l'acétylène après activation du biphényle par transfert de $\mathrm{H}$ par $\mathrm{H}$ :

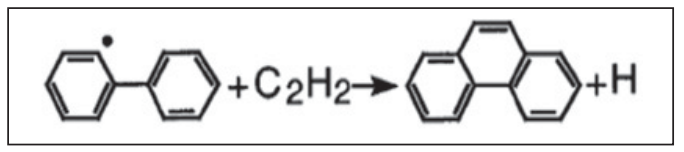

Marinov [25] et Castaldi [28] ont montré que la voie de formation des HAP, via la formation du benzène, était insuffisante pour rendre compte des concentrations des HAP formés. Les auteurs ont conclu que, 
dans les flammes de combustible aliphatiques dont celles de méthane, les HAP sont issus plutôt de la recombinaison des radicaux cyclopentadiényles qui conduit au naphtalène.

$$
\mathrm{O}+\mathrm{OO}+\mathrm{H}+\mathrm{H}
$$

Fascella et al. [29] ont proposé un mécanisme de formation des premiers HAP impliquant des espèces à un nombre pair d'atomes de carbone sans passer par le naphtalène. Selon ces auteurs, les premiers précurseurs des HAP sont le 1,3-butadiène et le radical phényle qui, après cyclisation, forment deux cycles en $\mathrm{C}_{10}$ ou $\mathrm{C}_{9}$ respectivement par élimination d'un atome d'hydrogène $\mathrm{H}$ ou d'un groupement méthyle :

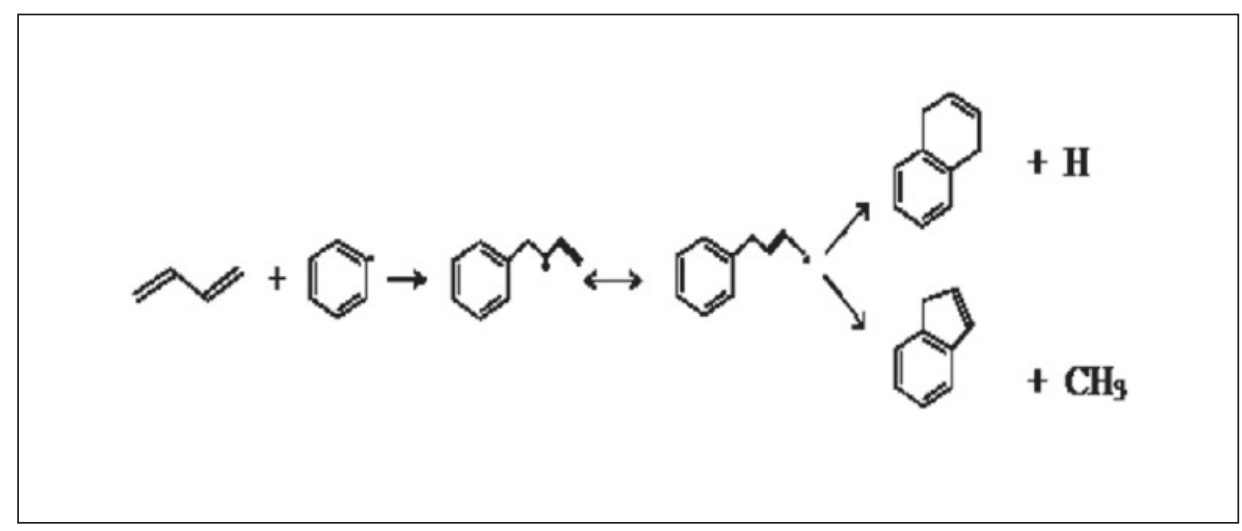

Un an plus tard, Fascella et al. [30] ont proposé une révision de leur mécanisme initial en postulant une formation des premiers HAP via des espèces à nombre de carbone impair. Ces espèces sont des radicaux en $\mathrm{C}_{5}$ et $\mathrm{C}_{7}$. La particularité de ce mécanisme, amorcé par l'addition de l'acétylène sur le radical cyclopentadiényle, réside dans le fait qu'il n'implique pas la formation du benzène. L'espèce centrale du mécanisme est le radical cyclo-heptatriényle $\left(\mathrm{cC}_{7} \mathrm{H}_{7}\right)$.

Une revue complète des avancées expérimentales et par modélisation sur la formation des HAP est disponible [31].

\section{LES MÉTHODES EXPÉRIMENTALES POUR L'ANALYSE DE STRUCTURE CHIMIQUE DE FLAMMES SUITÉES}

Dans ce chapitre, nous mentionnons les méthodes expérimentales les plus courantes utilisées pour la détection de HAP et de suies dans les flammes.

Trois types de techniques expérimentales peuvent être employés pour caractériser les HAP gazeux présents dans les flammes :

(1) Techniques analytiques de détection sélectives des espèces : c'est le cas des techniques chromatographiques (chromatographie en phase gazeuse (GC), chromatographie liquide à haute pression (HPLC), chromatographie à exclusion stérique (SEC)) et de spectrométrie de masse (SM). Couplées, ces techniques peuvent conduire à l'identification et au dosage des principaux HAP. Elles ont fourni l'essentiel des données quantitatives sur les HAP. Elles nécessitent un prélèvement préalable de l'échantillon dans la flamme au moyen d'une sonde [32].

(2) Techniques analytiques optiques non sélectives des espèces. Les HAP sont caractérisés par des spectres d'absorption très peu structurés à température ambiante s'étendant sur une large gamme spectrale. Il s'ensuit qu'une détection de HAP par des techniques comme l'absorption 


\section{EPJ Web of Conferences}

ou la fluorescence induite par laser (LIF) in situ dans la flamme n'est pas sélective du fait de la congestion des spectres d'absorption dans les conditions de pression et de température des flammes. Ce type de mesure peut cependant apporter des informations qualitatives sur des classes de taille de HAP et sur leur localisation par rapport aux particules de suie [33].

(3) Techniques optiques appliquées sur un échantillon refroidi après extraction par faisceau moléculaire. Dans ce cas les spectres d'absorption des HAP refroidis sont suffisamment résolus et structurés pour permettre une détection sélective. Deux méthodes sont applicables : la fluorescence induite par laser (jet-cooled LIF) [34] et l'ionisation multiphotonique résonante couplée à une analyse par spectrométrie de masse (REMPI) [35]. Ces techniques se développent depuis quelques années. Leur avantage par rapport aux premières (1) réside dans le fait qu'elles permettent d'accéder à des HAP de masse plus élevée et que leur sensibilité est plus grande.

La composition chimique des espèces adsorbées à la surface des suies est accessible après lavage avec un solvant approprié de la matière collectée dans les flammes, par GC ou HPLC [32]. Une méthode complémentaire, d'autant plus efficace que la masse des échantillons de suies est très faible, est la technique de désorption laser/ionisation laser/spectrométrie de masse [10, 32]. Elle peut être complétée par des analyses par FTIR ou Raman [36].

Pour ce qui concerne l'analyse des concentrations de suies, les méthodes les plus anciennes reposent soit sur la gravimétrie, c'est-à-dire consistent en une pesée des suies échantillonnées dans la flamme au moyen d'une sonde, soit sur l'extinction optique, c'est-à-dire que l'atténuation de l'intensité lumineuse au passage du panache de suies est directement liée à leur fraction volumique par l'intermédiaire de la loi de Beer-Lambert. Une variante de la technique d'extinction selon une traversée dans la flamme, est la technique cavity ring-down spectroscopy (CRDS) qui permet d'atteindre une excellente sensibilité [37]. Dans ces deux derniers cas, la mesure est intégrée spatialement selon le trajet du faisceau lumineux dans la flamme. Une alternative plus récente repose sur l'utilisation de l'incandescence induite par laser, méthode locale et très sensible. La technique d'Incandescence Induite par Laser (LII) consiste à porter les particules de suies présentes dans la flamme à très haute température sous l'action d'un rayonnement laser. L'analyse des radiations thermiques, de type corps noir (incandescence) permet de remonter à la fraction volumique de suies en valeur relative [33]. De plus la décroissance temporelle du signal d'incandescence est liée à la taille de la particule irradiée : plus la particule est grosse, plus elle met de temps à se refroidir. Sur le plan des distributions de tailles des particules, les méthodes les plus courantes reposent sur l'utilisation de méthodes couplées diffusion de la lumière/extinction [38] ou sur la technique SMPS (scanning mobility particle sizer) [7]. L'interprétation de ces méthodes granulométriques reste très délicate du fait de la morphologie très complexe des particules de suies. Les analyses fastidieuses de clichés TEM permettent probablement d'avoir les informations les plus fiables sur leur morphologie.

\section{Références}

[1] R.J. Kee, J.F. Grcar, M.D. Smooke, J.A. Miller, A fortran program for modelling steady laminar one-dimensional premixed flames, Sandia report SAND85-8240, 1985 et R.J. Kee, F.M. Rupley, J.A. Miller, Chemkin-II: A fortran chemical kinetics package for the analysis of gas phase chemical kinetics, Sandia report SAND89-8009B, 1989

[2] Dupont L., El Bakali A., Pauwels J.F. and Richter H., Combust. Flame, 135, pp 171-183, 2003.

[3] R.A. Dobbins, R.A. Fletcher, H.C. Chang, Combust. Flame 115 (1998) 285

[4] T. Ishiguro, Y. Takatori, K. Akihama, Combust. Flame 108 (1997) 231

[5] R.A. Dobbins, in: H. Bockhorn, A. D’Anna, A.F. Sarofim, H. Wang (Eds), “Combustion generated fine carbonaceous particles" (2009) Chapter 13, 189-204, KIT Scientific Publishing

[6] A. D’Alessio, A. D’Anna, P. Minutolo, L.A. Sgro, in: H. Bockhorn, A. D’Anna, A.F. Sarofim, H. Wang (Eds), "Combustion generated fine carbonaceous particles" (2009) Chapter 15, 231-246, KIT Scientific Publishing 
[7] B. Zhao, Z. Yang, M.V. Johnston, H. Wang, A.S. Wexler, M. Balthasar, M. Kraft, Combust. Flame 133 (2003), p. 173

[8] S.E. Stein, A. Fahr, J. Phys. Chem. A 89 (1985) 3714

[9] L.G. Blevins, R.A. Flechter, B.A. Benner, E.B. Steel, G.W. Mulholland, Proc. Combustion Institute 29 (2002) 2325

[10] Thesis A. Faccinetto, High sensitivity detection of Polycyclic Aromatic Hydrocarbons desorbed from soot particles using Laser Desorption/Laser Ionisation/Time-Of-Flight Mass Spectrometry. An approach for studying the soot growth process in flames, Université de Lille 1, 2009

[11] Richter H., Howard J.B., Progr. Energy Combust. Science, 26, 565, (2000).

[12] Frenklach M., Phys. Chem. Chem. Phys., 4, 2028-2037, (2002).

[13] Bockhorn H., Soot formation in combustion: mechanism and models. Springer, (1994).

[14] J. Appel, H. Bockhorn, M. Wulkow, Chemosphere 42 (2001) 635

[15] Chung and Violi, Carbon 45 (2007) 2400

[16] A. D’Anna in: H. Bockhorn, A. D’Anna, A.F. Sarofim, H. Wang (Eds), “Combustion generated fine carbonaceous particles" (2009) Chapter 19, 289-320, KIT Scientific Publishing

[17] O. Mathieu, N. Chaumeix, C. Paillard, F. Douce, Combust. Flame 156 (2009)1576

[18] M. Frenklach and S.J. Harris, J. Colloid Interface Sci. Vol 118, pp. 252, 1987.

[19] M. Frenklach and H. Wang, Detailed mechanism and modeling of soot particle formation, in Soot Formation in Combustion (Bockhorn, 1994), pp. 165

[20] H. Sabbah, L. Biennier, S.J. Klippenstein, I.R. Sims, B.R. Rowe, J. Phys. Chem Lett. 1 (2010) 2962

[21] Pope C.J., Howard J.B., Aerosol Sci. Technol., 27, 73-94, (1997).

[22] J. Z. Wen, M.J. Thomson, S.H. Park, S.N. Rogak, M.F. Lightstone, Proc. Comb. Institute 30 (2005) 1477

[23] K. Netzel, H. Lehtiniemi, F. Mauss, Proc. Comb. Institute 31 (2006) 667

[24] Miller J.A., Melius C.F., Combust. Flame, 91, 21, (1992).

[25] Marinov N., Pitz W.J., Westbrook C.K., Castaldi M., Senkan S.M., Combust Sci Technol, 116/117, 211, (1996).

[26] Frenklach M., Wang H., Combust. Flame, 87, 365-370, (1991).

[27] Bittner J.D., Howard J.B., Proc. Combust. Inst, 18, 1105, (1981).

[28] Castaldi M.J., Marinov N.M., Melius C.F., Huang J., Senkan S.M., Pitz W.J., Westbrook C.K., Proc. Comb. Institute 26 (1996) 693-702

[29] Fascella S., Cavallotti C., Rota R., Carrà S., J. Phys.Chem.A 108, 3829-3843 (2004).

[30] S. Fascella, C. Cavallotti, R. Rota, S. Carrà, J. Phys. Chem. A 109, 7546-7557 (2005).

[31] C.S. McEnally, L.D. Pfefferle, B. Atakan, K. Kohse-Höinghaus, Prog. Energy and Combust. Science, 32, (2006) 247

[32] B. Apicella, R. Barbella, Ciajolo, A. Tregrossi, Combust Sci Technol 174 (2002) 309

[33] Lemaire R., Faccinetto A., Therssen E., Ziskind M., Focsa C., Desgroux P., Proc. Comb. Institute 32 (2009) 737-744

[34] Mercier X., Wartel M., Pauwels J.F., Desgroux P., Appl. Phys. B 91, 387-395, 2008

[35] Y. Carpentier, "De la flamme de laboratoire au milieu interstellaire: signatures spectrales d'entités aromatiques libres et assemblées, Thèse Université Paris XI (2009)

[36] J.P. Cain, P.L. Gassman, H. Wang, A. Laskin, PCCP 12 (2010) 5206

[37] X. Mercier, P. Desgroux, in: G. Berden, R. Engeln (Eds.), Cavity Ring-Down Spectroscopy: Techniques and Applications. A John Wiley and Sons, Inc., Publication, 2009, Chapt. 10, pp. 273-311

[38] P. Van-Hulle, M. Talbaut, M. Weill, A. Coppalle, Meas. Sci. Technol. 13 (2002) 375 